\title{
Permeability of Dual-Structured Porous Media
}

\author{
Ehsan Khajeh" and Daan M. Maijer
}

Department of Materials Engineering, The University of British Columbia, 6350 Stores Road, Vancouver, BC, V6T 1Z4, Canada

\begin{abstract}
The effective permeability of dual-structured porous media has been determined through physical and numerical modeling. Example porous media consisting of a woven network of cylindrical bars overlaid by a network of spherical particles have been produced by a rapid prototyping technique. A glycerin-based solution, held in a pressurized reservoir, was forced through the porous samples. The resulting flow rate and pressure drop across the sample were measured and used to determine the permeability of each sample. Mathematical models, considering the continuity and momentum equations, were also developed for the geometries corresponding to the physical models. The results were compared with the analytical expressions of permeability reported by Khajeh and Maijer, Liu et al., and Carman-Kozeny. The measured permeabilities are in better agreement with the expressions reported by Khajeh and Maijer and Liu et al. than those calculated with the Carman-Kozeny expression.
\end{abstract}

Keywords: Permeability, dual-structured, porous media, physical modeling, numerical modeling.

\section{INTRODUCTION}

Characterizing the flow conductance, namely the permeability, is one of the most important parameters, when investigating mass-transport phenomena in saturated porous media. For porous media that exhibit a variety of heterogeneities in their structures, the effective permeability may be described by using dual-structured model. In such model, the medium is considered to be made up of two regions, one associated with macro-pore or fractured network and the other filled within the void space of the fractured network [1].

For an idealized dual-structured porous medium, Liu et al., [2] introduced a theoretical expression, based on an analytical solution [3] to the Brinkman-Darcy equation, to describe the effective permeability of an array of square impermeable blocks placed in a fluid saturated porous medium. They focused on an equivalent continuum model and proposed an analytical expression to determine the effective permeability. Sano et al., [4] extended Liu et al.'s [2] work to calculate the effective permeability of a porous medium with impermeable blocks of different sizes. Khajeh and Maijer [5] developed an analytical expression based on a solution to the Brinkman-Darcy equation for the permeability of solidifying hypoeutectic aluminum alloys by considering the microstructure to be a dual-structured porous medium. The results of this study [5] showed that the expression developed based on solution to the BrinkmanDarcy equation is applicable to non-idealized dual-structured porous media.

In this study, the theoretical expression developed by Khajeh and Maijer [5] will be presented in a more general form. To assess the performance of the expression, the

*Address correspondence to this author at the Department of Materials Engineering, The University of British Columbia, 309-6350 Store Road, Vancouver, BC, V6T1Z4, Canada; Tel: +1 (604) 822-6013; Fax: +1 (604) 822-3619;

E-mails: ehsan.khajeh@gmail.com, ekhajeh@interchange.ubc.ca effective permeability of example dual-structured porous media consisting of a network of impinged spheres and woven bars is characterized through physical and numerical modeling and compared with predictions from various permeability expressions.

\section{ANALYTICAL EXPRESSION}

Khajeh and Maijer [5] developed an analytical expression for the effective permeability of a dual-structured porous medium. In this work, Porous Medium 1 is a network of impinged spheres with diameters of $D_{1}$ and porosity of $\varepsilon_{l}$, that is overlaid on Porous Medium 2 which is a network of interwoven bars with porosity of $\varepsilon_{2}$ and permeability of $K_{2}$. The porosity of each porous medium is calculated based on the volume of pores, or void space, per unit volume of the medium. Based on the solution to the Brinkman-Darcy equation and assuming that the empirical Carman-Kozeny equation for packed beds [6],

$$
K=\frac{\varepsilon^{3} D^{2}}{180(1-\varepsilon)^{2}}
$$

is valid to describe the permeability of a porous medium consisting of a network of impinged spheres with uniform diameter, the effective permeability of the dual-structure medium is given by:

$$
K_{e}=\frac{\varepsilon_{2} \varepsilon_{1}^{3} D_{1}^{2}}{60\left(1-\varepsilon_{1}\right)^{2}} G(\theta)
$$

where

$$
G(\theta)=\frac{1}{\theta^{2}}-\frac{\tanh (\theta)}{\theta^{3}}
$$


$\theta=\frac{\varepsilon_{1} D_{1}}{\sqrt{60}\left(1-\varepsilon_{1}\right)} \sqrt{\frac{\varepsilon_{2}}{K_{2}}}$

For the limiting case where there is no Porous Medium 2 (the void space of Porous Medium 1 is filled with fluid), $\varepsilon_{2-}$ $\rightarrow 1, K_{2} \rightarrow \infty$, and $\theta \rightarrow 0$. For this case, $G(\theta) \rightarrow 1 / 3$ and $K_{e}$ returns equation (1). For the other limiting case where there is no Porous Medium $1, \varepsilon_{l} \rightarrow 1, \theta \rightarrow \infty$ and $G(\theta) \rightarrow 1 / \theta^{2}$. For this case, $K_{e}$ returns $K_{2}$. These limiting cases verify the performance of equation (2) with known behavior at the extremes of the dual-structure geometric conditions. For the case where the permeability of Porous Medium 1 is much larger than the permeability of Porous Medium 2, $\theta$ is large, $G(\theta) \rightarrow 1 / \theta^{2}$ and $K_{e}=\varepsilon_{1} K_{2}$. This suggests that the effective permeability is determined predominately by the permeability of the finer porous medium, i.e. Porous Medium 2. This is in agreement with the expression developed by Gerke and Van-Genuchten [1] to describe saturated water flow in a structured soil system (micro scale soil in a macro scale rock matrix). Assuming no fracture in the rock, the problem is physically similar to the case of large $\theta$.

After verifying the current mathematical expression with limiting cases, the permeability for example dual-structured porous media has been determined and compared with predictions from equation (2) and other expressions.

\section{PERMEABILITY DETERMINATION}

\subsection{Porous Samples}

Porous samples consisting of a network of impinged spheres and woven bars have been developed for this study.

The network of woven bars, consisting of two sets of $7 \times 7 \times 7$ bars offset by $1 / 2$ the inter-bar spacing in each direction, form a porous cube with a porosity fraction of 0.45 and an edge length of $50 \mathrm{~mm}$ (refer to Fig. 1-a). To generate the second overlaid porous medium, random locations were chosen within the network of woven bars as the origins of spherical particles and a Cellular Automaton (CA) model was employed to simulate their growth. For the sake of brevity, the reader is referred to [7] for a detailed description and assessment of the CA model employed in this study. The CA model was used to generate porous media with fractions of porosity, or void space, varying from 0.45 to 0.1 and different densities of spherical particles $(6,50$ and 90 /domain). An example of the simulated porous medium produced with 50 spherical particles representing various porosity fractions are shown in Fig. (1-b to d).

\subsection{Physical Determination of Permeability}

To measure the permeability of the dual-structured porous geometries, a physical modeling approach has been employed. Physical models of the porous geometries described in section 3.1 with 50 spherical particles and porosity fractions of $0.45,0.36,0.26$, and 0.18 were constructed by Selective Laser Sintering (SLS). The SLS rapid prototyping machine used to produce the samples for this study was capable of an accuracy of $0.15 \mathrm{~mm}$. Fig. (2) shows an example of a constructed replica for 50 spherical particles and porosity fraction of 0.26 . The permeability of each replica was measured by passing glycerin through the physical models and measuring the discharge flow rate and pressure drop. The permeability was then calculated using Darcy's Law:

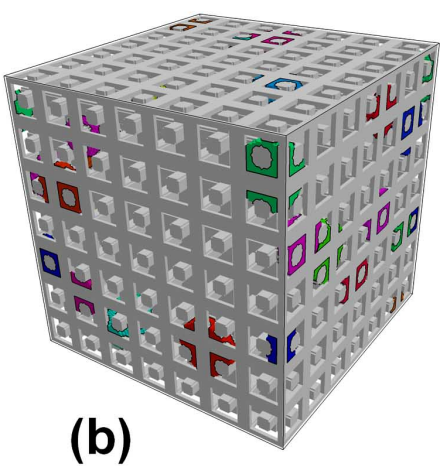

(a)
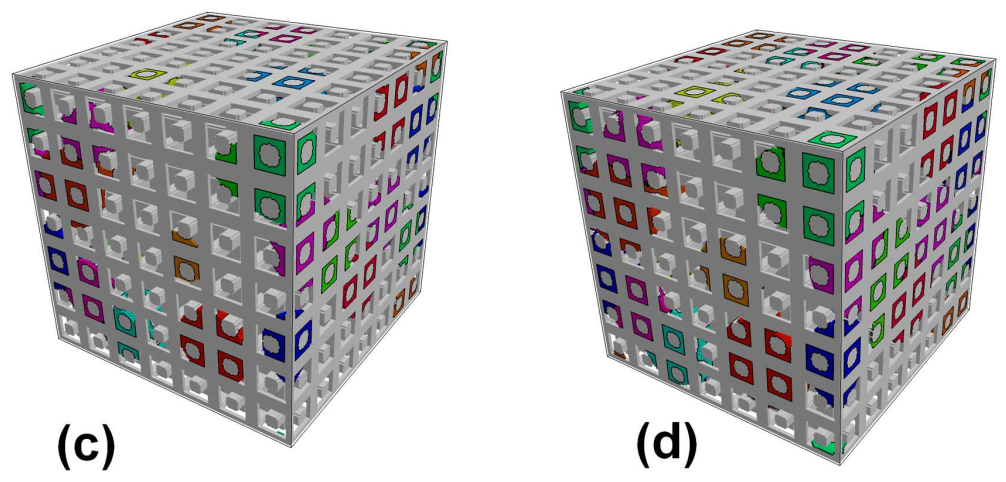

Fig. (1). Dual-structured porous media with $N=50$ and (a) $\varepsilon=0.45$, (b) $\varepsilon=0.36$, (c) $\varepsilon=0.26$, and (d) $\varepsilon=0.18$. 


$$
K=\frac{q \mu L}{\Delta P}
$$

where $K$ is the permeability, $q$ is the superficial velocity, $\mu$ is the viscosity of glycerin, $\Delta P$ is the pressure drop across the replica, and $L$ is the length of the sample. A detailed description of the apparatus and testing procedure used in this study can be found in [8].

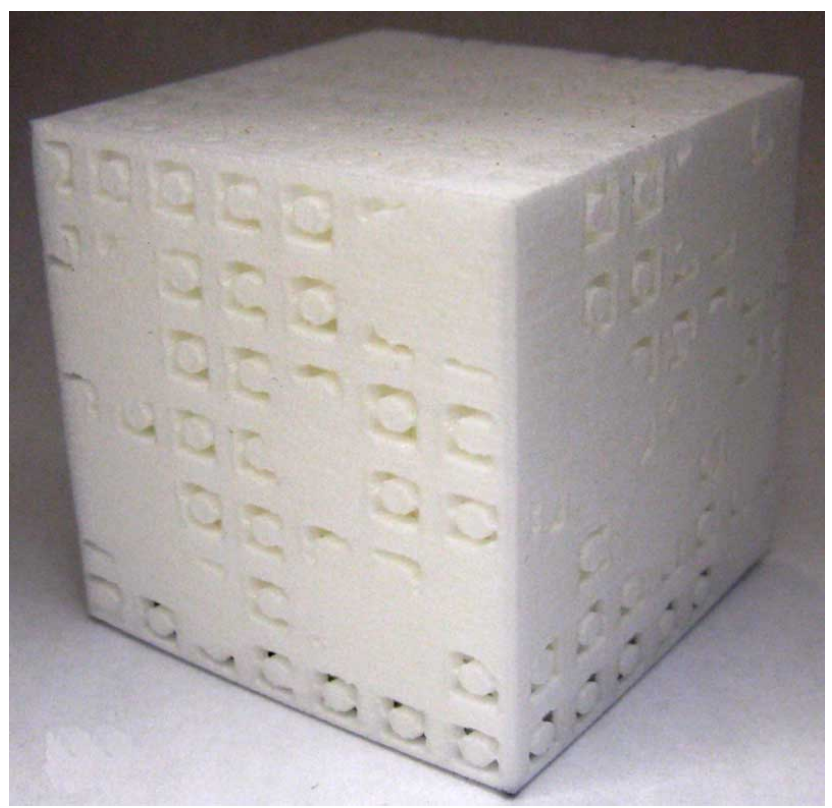

Fig. (2). SLS replica of the porous medium with $N=50$ and $\varepsilon=0.26$ (refer to Fig. 1-c).

The permeability tensor was measured by sequentially reorienting the replica samples inside the apparatus. To assess the consistency of the measurements, 4-10 successive measurements of the pressure and the flow rate were performed for each replica sample orientation.

\subsection{Numerical Determination of Permeability}

The permeability of the porous samples with 6,50 , and 90 spherical particles (described in section 3.1) was determined numerically by solving the full incompressible Navier-Stokes and continuity equations:

$\rho\left(\frac{\partial u}{\partial t}+u \cdot \nabla u\right)=-\nabla p+\mu \nabla^{2} u$

$\nabla \cdot u=0$

where $p$ and $u$ are the local pressure and velocity in the computational domain, $\rho$ is the liquid density $\left(1260 \mathrm{~kg} \mathrm{~m}^{-3}\right)$, and $\mu$ is the viscosity (1.2 $\mathrm{Pa} \mathrm{s})$. The permeability tensor for the cubic domain was calculated through sequential runs of the model where a low velocity flow was applied in the directions normal to each face.

The flow problem was solved with a $2^{\text {nd }}$-order accurate Finite Volume Method (FVM) using the commercial CFD software, ANSYS CFX (ANSYS and CFX are registered trademarks of ANSYS, Inc., USA). A detailed description of the modeling methodology used in this study is available in
[9]. An example of the predicted pressure distribution used in the calculation of the permeability is shown in Fig. (3). Using the predicted pressure drop across the domain and the superficial velocity of the fluid, the permeability was calculated using equation (5).

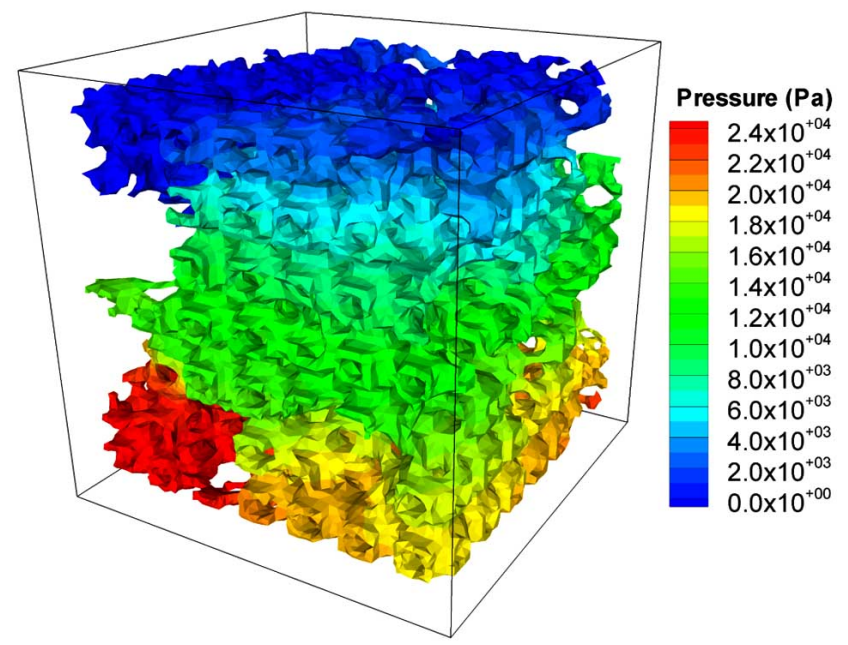

Fig. (3). Simulated pressure contour of porous medium with $N=50$ and $\varepsilon=0.26$ (refer to Fig. 1-c).

\section{RESULTS AND DISCUSSION}

The permeabilities (average of $K_{x x}, K_{y y}$ and $K_{z z}$ ) characterized experimentally and numerically are plotted versus the porosity fraction in Fig. (4). Numerical results are given for

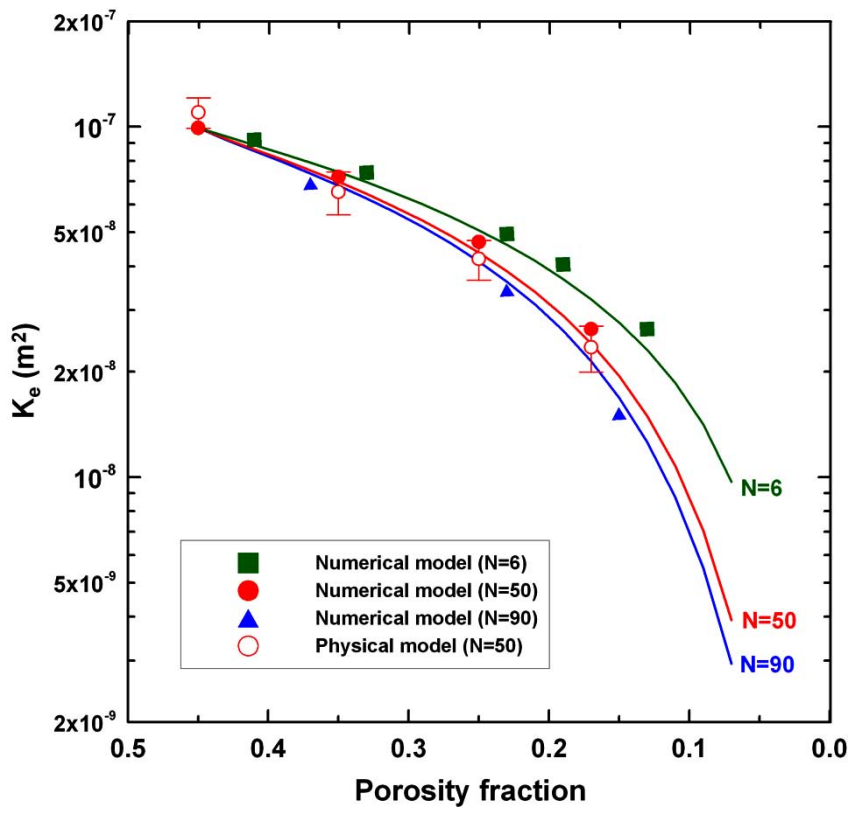

Fig. (4). Effective permeability versus porosity fraction. Closed and open symbols represent numerical and physical permeabilities, respectively. Lines indicate permeability based on the equation (2).

geometric conditions with the number of spherical particles, $N$, equal to 6,50 and 90 . As shown, the absolute values of permeability decrease as $N$ increases and porosity fraction decreases. The numerically calculated permeabilities for $N$ 
equal to 50 are in close agreement with the permeabilities measured on the physical models (within $\pm 20 \%$ of those determined through measurement). The differences between the numerically calculated and experimentally measured permeabilities can be attributed to the measurement uncertainties as discussed in [8]. The comparison of results for $N$ equal to 50 suggests that the numerical modeling techniques employed in this study provide a good representation of the physics of this problem. Thus, the results for $N$ equal to 6 and 90 are expected to be accurate representations of the variation of permeability for these structures. Fig. (4) also presents the permeabilities calculated with equation (2). As shown, the evolution of permeability with porosity fraction described by equation (2) is in good agreement with the numerically calculated values of permeability.

\subsection{Characterized Permeabilities versus Available Expressions}

To analyze the performance of the analytical expressions in more detail, the numerically predicted permeabilities have been compared with permeabilities calculated through equation (2) and two other expressions. Fig. (5) shows the numerically characterized permeabilities (normalized with the solid/liquid interfacial area per unit volume of solid, $S_{v}$ ) versus the total porosity fraction, $\varepsilon\left(\varepsilon=\varepsilon_{I} \varepsilon_{2}\right)$. For comparison, lines representing equation (2), Liu et al.'s expression [2]:

$$
K_{e}=\frac{\left(1-\left(1-\varepsilon_{1}\right)^{1 / 3}\right)^{2}\left(1-\left(1-\varepsilon_{1}\right)^{2 / 3}\right) \varepsilon_{2} D_{1}^{2}}{4\left(1-\varepsilon_{1}\right)^{2 / 3}} G(\theta)
$$

where

$$
\theta=\frac{1-\left(1-\varepsilon_{1}\right)^{1 / 3} D_{1}}{2\left(1-\varepsilon_{1}\right)^{1 / 3}} \sqrt{\frac{\varepsilon_{2}}{K_{2}}}
$$

, and the Carman-Kozeny expression [10]:

$$
K=\frac{\varepsilon^{3}}{k_{C} S_{v}^{2}(1-\varepsilon)^{2}}
$$

where $S_{v}$ is the solid/porosity interfacial area per unit volume of solid and $k_{C}$ is a constant, have been plotted in Fig. (5). The evolution of permeability with porosity fraction described by equation (2) is in good agreement with the numerically calculated values of permeability for the range of $N$ studied. Permeabilities calculated from the CarmanKozeny expression deviate from the characterized permeabilities as $N$ increases. The smaller deviation with smaller $N$ is equivalent to the previous described case of large $\theta$ which leads to $K_{e} \rightarrow \varepsilon_{1} K_{2}$. The deviation of the permeability calculated with Liu et al.'s expression [2] from the numerically predicted permeabilities decreases as $N$ increases. This suggests that Liu et al.'s expression should only be used for predicting the permeability when the length scales of two porous media are close, where as equation (2) can be used for a wide variety of length scales.

\subsection{Comparison with Related Studies}

Sano et al., [4] measured the effective permeability of fluid saturated porous media consisting of small and large
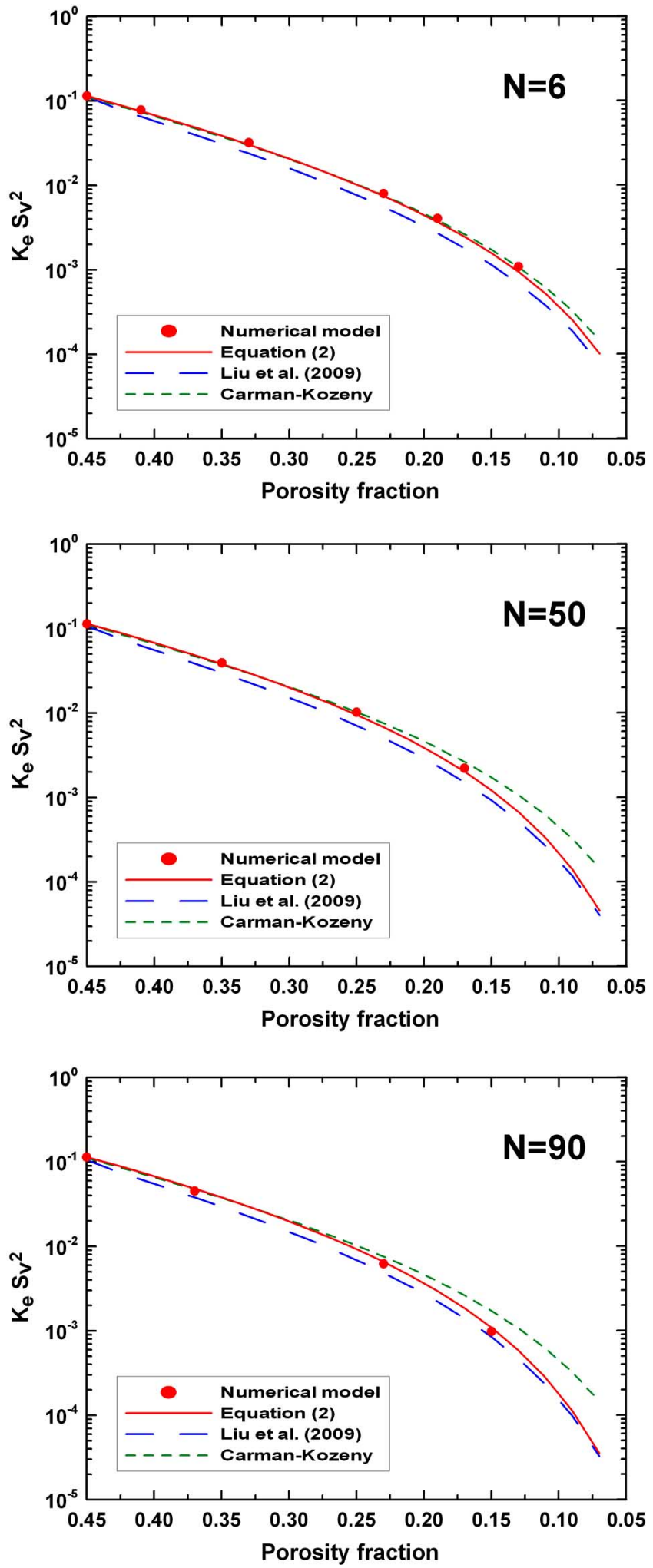

Fig. (5). Dimensionless permeability versus porosity fraction. Symbols represent numerical permeabilities. Lines indicate calculated permeabilities from equation (2), (8) and (10).

spherical particles. Table 1 shows their measured permeabilities compared with permeabilities calculated using the 
Table 1. Permeability of Porous Media Consisting of Spherical Particles of Different Sizes

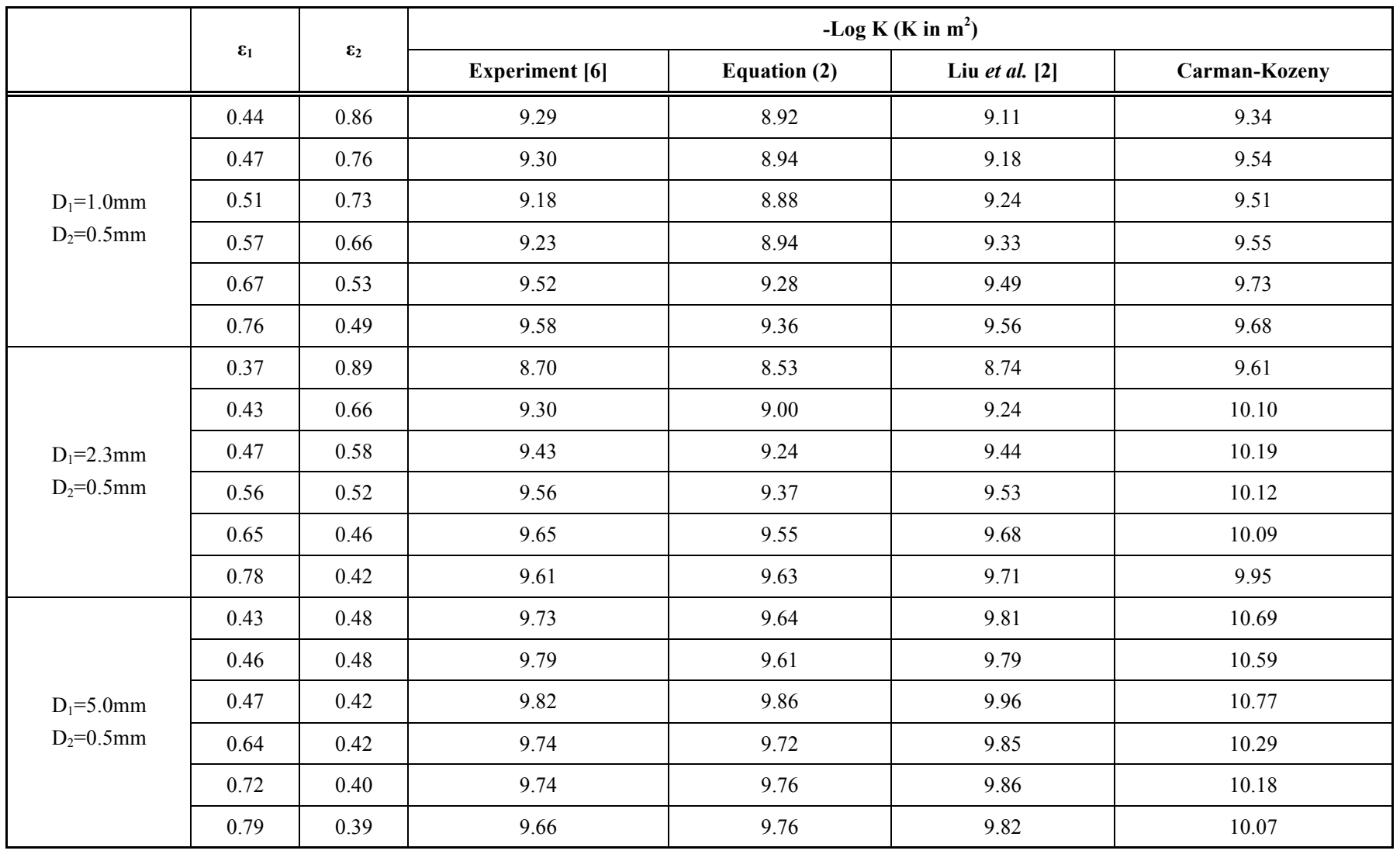

current mathematical expression (refer to equation (2)), Liu et al.'s expression [2] (refer to equation (8)), and the Carman-Kozeny expression for packed beds [6] (refer to equation 1)). The permeability calculated using expressions derived from the solution of Brinkman-Darcy equation, i.e. equation (2) and Liu et al.'s, are in a closer agreement with experimental values than those calculated by the CarmanKozeny expression. Moreover, the Liu et al.'s permeabilities deviate from experimental values as the difference between length scales of two porous media, i.e. diameters of particles, increases. This is in accordance with the results of this study as discussed in the previous section.

\section{CONCLUSIONS}

The effective permeability of example dual-structured porous media has been determined through physical and numerical modeling and compared with the permeability calculated by three available analytical expressions. Comparison of the numerically calculated and measured permeabilities showed close agreement (within $\pm 20 \%$ ). Studying the characterized permeabilities of dual-structured porous media in this study and in Sano et al.'s study showed that expressions derived from the solution of Brinkman-Darcy equation, i.e. equation (2) and Liu et al.'s, are in a closer agreement with experimental values than those calculated with the Carman-Kozeny expression. Hence, these mathematical models can be used for estimating the permeability of dualstructured porous media.

\section{ACKNOWLEDGEMENT}

The authors acknowledge the Natural Sciences and Engineering Research Council of Canada (NSERC) for research support.

\section{CONFLICTS OF INTEREST}

The authors declare no conflict of interest.

\section{REFERENCES}

[1] H.H. Gerke and M.T. Vangenuchten, "A dual-porosity model for simulating the preferential movement of water and solutes in structured porous-media", Water Resources Research, vol. 29, pp. 305-319, Feb 1993.

[2] J. Liu, Y. Sano, and A. Nakayama, "A simple mathematical model for determining the equivalent permeability of fractured porous media," International Communications in Heat and Mass Transfer, vol. 36, pp. 220-224, 2009.

[3] A. Nakayama, H. Koyama, and F. Kuwahara, "An analysis on forced convection in a channel filled with a Brinkman-Darcy porous medium - Exact and approximate solutions," Warme Und Stoffubertragung-Thermo and Fluid Dynamics, vol. 23, pp. 291 295, 1988.

[4] Y. Sano, K. Noguchi, and T. Kuroiwa, "An experimental investigation into the effective permeability of porous media whose matrices are composed of obstacles of different sizes", The Open Transport Phenomena Journal, vol. 1, pp. 15-19, 2009.

[5] E. Khajeh and D. M. Maijer, "Permeability of dual structured hypoeutectic aluminum alloys", Acta Materialia, vol. 59, pp. 45114524, Jun. 2011.

[6] F. A. L. Dullien, Porous Media: Fluid Transport and Pore Structure. New York: Academic Press, 1979. 
[7] E. Khajeh and D.M. Maijer, "Inverse Analysis of Eutectic Nucleation and Growth Kinetics in Hypoeutectic Al-Cu Alloys", Metallurgical and Materials Transactions a-Physical Metallurgy and Materials Science, vol. 42A, pp. 158-169, Jan. 2011.

[8] E. Khajeh and D.M. Maijer, "Physical and numerical characterization of the near-eutectic permeability of aluminumcopper alloys", Acta Materialia, vol. 58, pp. 6334-6344, Nov. 2010 .
[9] E. Khajeh and D. M. Maijer, "Numerical determination of permeability of $\mathrm{Al}-\mathrm{Cu}$ alloys using 3D geometry from X-ray microtomography", Materials Science and Technology, vol. 26, pp. 1469-1476, Dec 2010.

[10] P. C. Carman, Flow of gases through porous media. London, UK: Butterworth Scientific, 1956.

(C) Khajeh and Maijer; Licensee Bentham Open.

This is an open access article licensed under the terms of the Creative Commons Attribution Non-Commercial License (http://creativecommons.org/ licenses/by-nc/3.0/), which permits unrestricted, non-commercial use, distribution and reproduction in any medium, provided the work is properly cited. 